\title{
Integrales elípticas en la solución del péndulo no lineal
}

\section{Elliptic Integrals in the Solution of the Non-Linear Pendulum}

\author{
Sergio Herrera \\ Docente de planta Programa de Física \\ Grupo de física teórica \\ Universidad Surcolombiana \\ Anyery Dussán \\ Estudiante de pregrado Programa de Física \\ Grupo de física teórica, semillero Peter Higgs \\ Universidad Surcolombiana. \\ Francis Segovia \\ francis.segovia@usco.edu.co
}

\section{Resumen}

En este trabajo presentamos la solución de la ecuación diferencial de segundo orden no lineal para el péndulo simple. Este problema puede ser utilizado para introducir conceptos de integrales elípticas y motivar así al estudiante el uso de software computacional para analizar las soluciones obtenidas, tomamos como referencia el trabajo de Beléndez para hallar las soluciones. Las soluciones obtenidas para el desplazamiento y velocidad angular como función del tiempo se encuentra en términos de las funciones elípticas de Jacobi $s n(u ; m)$.

Palabras clave: Desplazamiento angular, integrales elípticas, leyes de Newton, péndulo simple, periodo.

\begin{abstract}
In this paper we present the solution to the second level non-linear differential equation for the simple pendulum. This problem may be sued to introduce concepts of elliptic integrals and thus motivate the student in the use of computational software to analyze the obtained solutions, we take as reference the work of Beléndez to find the solutions. The solutions obtained for the displacement and angular velocity as a function of time is found in terms of the elliptic functions of Jacobisn (u;m).
\end{abstract}

Key Words: Angular displacement, elliptic integrals, Laws of Newton, simple pendulum, angular period.

\section{Introducción}

Los movimientos periódicos se caracterizan que después de transcurrido cierto tiempo llamado periodo, el sistema vuelve a su posición y velocidad inicial, después de lo cual el movimiento se repite una y otra vez. Por tanto, el movimiento ocurre en ciclos repetitivos, cada uno de los cuales es exactamente igual a cualquier otro (Alonso y Finn, 1970). Por ejemplo, la Tierra sigue esencialmente la misma órbita elíptica alrededor del sol, año tras año. Los movimientos periódicos ocurren frecuentemente en la naturaleza y ocupan un lugar muy importante en la física. Los que pueden describirse en función de una sola coordenada de distancia como el movimiento ascendente y descendente de una masa suspendida de un resorte vertical, se llaman movimientos oscilatorios. El latido del corazón, la vibración de una cuerda de violín, el balanceo de un péndulo y la pulsación de los átomos en un cristal, son ejemplos de movimientos oscilatorios (McKelvey, 1981).

Hay muchos tipos de movimientos oscilatorios algunos de los cuales son sumamente complejos. Empero, hay una forma que se encuentra con mucha frecuencia y que también es muy simple: el movimiento armónico simple. El movimiento armónico simple ocurre siempre que la fuerza resultante que actúa en un objeto tiene dirección opuesta y es directamente proporcional a su desplazamiento, a partir de una posición de equilibrio en el que la fuerza resultante es cero. 


\section{Metodología}

En el presente trabajo estudiaremos el péndulo simple, ilustrado en la figura 1. En el problema planteado se supone que una pequeña masa $m$ está suspendida de una cuerda de longitud $l$ y masa despreciable, fija a un soporte superior estacionario. Se supone que la masa es tan pequeña que su comportamiento es esencialmente de una masa puntual.

Figura 1. Péndulo simple.

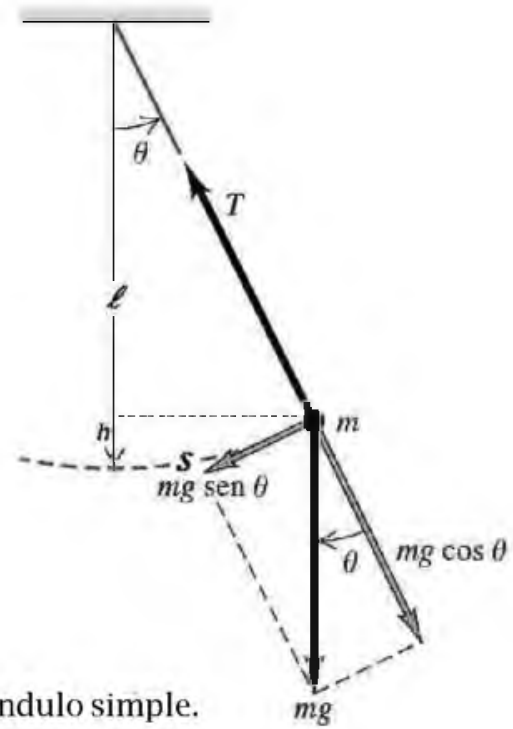

En la figura anterior se representa al péndulo en alguna posición arbitraria de su ciclo donde la cuerda forma un ángulo $\theta$ con la posición de equilibrio, es $\theta$ decir representa desplazamiento angular del péndulo. Las únicas fuerzas que actúan sobre la masa son el peso $m g$ (que se indica descompuesto en su componente paralela y normal a la cuerda) y la tensión $\vec{T}$. La única componente de fuerza que genera momento, es la componente del peso $m g \sin (\theta)$ paralela a la trayectoria sobre la que se mueve la masa (Serway, 2009).

Aplicando la segunda ley al sistema descrito anteriormente, se obtiene una ecuación diferencial de segundo orden,

$$
\frac{d^{2} \theta}{d t^{2}}+w_{0}^{2} \sin \theta=0
$$

En la ecuación (1) $\mathrm{w}_{0}$ representa la frecuencia angular natural de oscilación dada por:

$$
w_{0}=\sqrt{\frac{8}{1}}
$$

Donde g representa la aceleración de la gravedad. En este trabajo resolveremos la ecuación (1) en el caso en que el desplazamiento angular es $» 1 \mathrm{rad}$ (o sea, $» 5.3^{\circ}$ ). Lo anterior implica que generalmente la aproximación que se realiza en la literatura $\sin \theta \subseteq \theta$ no es válida. Siguiendo el trabajo de Beléndez (2007), impondremos las siguientes condiciones iniciales para la ecuación de movimiento:

$$
\theta(t=0)=\theta_{0},\left.\quad\left(\frac{d \theta}{d t}\right)\right|_{t=0}=0
$$

En la ecuación (3) $\theta_{0}$ es el desplazamiento angular inicial. Restringiremos nuestro estudio al no considerar fuerzas disipativas (de rozamiento), esto hace que la energía mecánica total se conserve, dando como resultado que el péndulo oscile en el intervalo $\left[-\theta_{0,}+\theta_{0}\right]$

Multiplicamos la ecuación (1) por $\frac{\text { de }}{\text { iff }}$

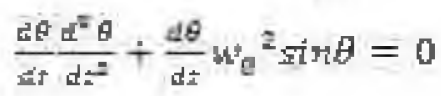

Que se reescribe así

$$
\frac{d}{d t}\left[\frac{1}{2}\left(\frac{d e^{2}}{d y^{2}}\right)^{2}-w_{0}^{2} \cos \theta\right]
$$

La ecuación (5) hace referencia a la conservación de la energía mecánica total, al integrarla y aplicar las condiciones iniciales dadas por la ecuación (3), obtenemos

$$
\frac{1}{2}\left(\frac{d \theta}{d t}\right)^{2}-w_{0}^{2} \cos \theta=-w_{0}^{2} \cos \theta_{0}
$$

Teniendo en cuenta la identidad trigonométrica $\cos \theta=1-2 \sin ^{3}\left(\frac{9}{2}\right)$, la ecuación anterior toma la siguiente forma:

$$
\left(\frac{d \theta}{d E}\right)^{2}=4 w_{0}^{2}\left[\sin ^{2}\left(\frac{\theta_{0}}{2}\right)-\sin ^{2}\left(\frac{\theta}{2}\right)\right]
$$

Haciendo los siguientes cambios de variable

$$
\sin ^{2}\left(\frac{\theta_{0}}{2}\right)=k \quad \text { y } \sin \left(\frac{\theta}{2}\right)=y \text {, fácilmente }
$$

se demuestra que de las condiciones iniciales dadas por la ecuación (3), obtenemos la siguiente relación:

$y(t=0)=\sqrt{k} \quad$ y $\quad \frac{d y}{d t}=\frac{d y}{d \theta} \frac{d \theta}{d t}=\frac{1}{2} \cos \left(\frac{\theta}{2}\right) \frac{d \theta}{d t}$

Debe tenerse en cuenta $\theta$ varía entre 0 y $\pi$, por lo tanto, $k$ está en el intervalo $0<k<1$, al elevar al cuadrado la ecuación (8) se obtiene:

$$
\left(\frac{d e}{d x}\right)^{2}=\frac{4}{1-y^{2}}\left(\frac{d y}{d x}\right)^{2}
$$

La ecuación (9) anterior ecuación se expresa ahora en términos de las nuevas variables $k$ y $y$, así:

$$
\left(\frac{d y}{d t}\right)^{z}=w_{0}{ }^{2} k\left(1-y^{2}\right)\left(1-\frac{y^{2}}{k}\right)
$$


Considerando las siguientes variables adimensionales,

$$
\tau=w_{0} t, z=\frac{y}{\sqrt{t}}
$$

De las condiciones dadas por la ecuación (8), la nueva variable $z$ satisface:

$$
z(\tau=0)=1 \quad \text { y }\left.\left(\frac{i z}{\bar{s}}\right)\right|_{\tau=0}=0
$$

Al reemplazar la ecuaciones (11) en la ecuación (10), esta última toma la siguiente forma:

$$
\left(\frac{d z}{d z}\right)^{2}=\left(1-z^{2} k\right)\left(1-z^{2}\right)
$$

Despejamos $d \tau$ de la ecuación (13)

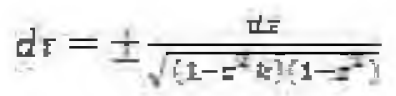

Escogemos el signo negativo ya que se está trabajando en la parte de abajo del plano, haciendo el cambio

$$
z \rightarrow \gamma \text { donde } \tau \in[0, i] \text { y } Y \in[1, z]
$$

Al resolver la integral se demuestra fácil mente,

$$
\tau=\int_{0}^{1} \frac{d y}{\sqrt{\left(1-r^{2} k\right)\left(1-r^{2}\right)}}-\int_{0}^{z} \frac{d y}{\sqrt{\left(1-r^{2} k\right)\left(1-r^{2}\right)}}
$$

Definiendo las integrales elípticas (Abramowitz y Stegun, 1972):

$$
\begin{aligned}
& K(m)=\int_{0}^{1} \frac{d z}{\sqrt{\left(1-x^{2}\right)\left(1-m x^{2}\right)}} \quad y \\
& F(\varphi ; m)=\int_{0}^{\varphi} \frac{d z}{\sqrt{\left(1-z^{2}\right)\left(1-m x^{1}\right)}}
\end{aligned}
$$

Haciendo $z=\$$ in ciones anteriores, la ecuación (15) toma la forma

$$
\tau(z)=k(k)-F(\operatorname{Arcsin}(z) ; k)
$$

La anterior ecuación se puede expresar en términos de la función elíptica de Jacobi $s n(u ; m)$ así:

$$
\Sigma=m(k(k)-r ; k)
$$

Finalmente, recordando la ecuación (11) y además que $\sin ^{2}\left(\frac{\theta_{a}}{2}\right)=\not h$ y $\sin \left(\frac{\theta}{2}\right)=y$, de la ecuación(18) se demuestra que el desplazamiento angular es:

$$
\begin{array}{r}
\theta(t)=2 \operatorname{Arcsin}\left[\operatorname { s i n } ( \frac { \theta _ { 0 } } { 2 } ) \operatorname { s n } \left[K\left(\sin ^{2} \frac{\theta_{0}}{z}\right)\right.\right. \\
\left.\left.-w_{0} t ; \sin ^{2} \frac{\theta_{0}}{2}\right]\right]
\end{array}
$$

Una importante relación en el estudio del péndulo simple es la energía mecánica, para nuestro caso podemos distinguir que la energía rotacional viene dada por:

$$
E_{R}=\frac{1}{2} I \phi^{2}
$$

Siendo $l$ el momento de inercia dado por $l=m l^{2}$

y $\phi\left(=\frac{d \theta}{d t}\right)$ es la velocidad angular del cuerpo.

La energía potencial gravitatoria, teniendo en cuenta la altura h del sistema descrito por la figura 1, es:

$$
B_{p}=m g h=m g l(1-\cos \theta)
$$

La energía mecánica total es la suma de las energías dadas por las ecuaciones (20) y (21),

$$
E=E_{\mathrm{p}}+E_{\mathrm{F}}=\frac{1}{2} m l^{2} \phi^{2}+m g l(1-\cos \theta)
$$

Debido al principio de conservación de la energía y por la condición impuesta en el problema presente al no tratar fuerzas disipativas, la ecuación (22) debe ser válida para cualquier instante de tiempo,

$$
\begin{aligned}
& \frac{1}{2} m l^{2} \phi\left(l^{2}+m g l[1-\cos \theta(t)]\right. \\
& =\frac{1}{2} m l^{2} \phi_{0}{ }^{2}+m g l\left(1-\cos \theta_{0}\right)
\end{aligned}
$$

De la anterior ecuación, obtenemos la expresión exacta de la velocidad angular en función del tiempo, donde $G(t)$ el desplazamiento angular viene dado por la ecuacion (19).

$$
\phi(t)= \pm \sqrt{2 \omega_{0}\left[\cos \theta(t)-\cos \theta_{0}\right]+\phi_{0}{ }^{2}}
$$

\section{Resultados}

Haciendo uso del resultado obtenido por la ecuación (19), en la figura 2 se representa el desplazamiento angular cuando el desplazamiento angular inicial aumenta para cuatro valores diferentes.

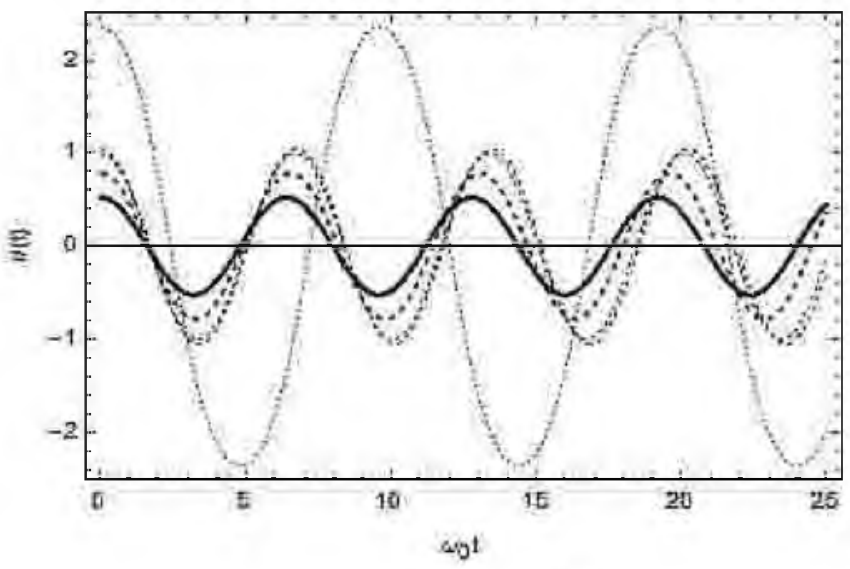

Figura 2. Desplazamiento angular $\theta$ dado por la ecuación (19), en función de $w_{0} t$. Se eligieron diferentes desplazamientos iniciales de $\theta_{0}=30^{\circ}, 45^{\circ}, 57.33^{\circ}, 60^{\circ}$ y $135^{\circ}$ 
En la figura 2, se observa que el desplazamiento angular aumenta en función del desplazamiento inicial. Al aumentar la amplitud de un péndulo simple se llega a un punto en que el movimiento angular deja de ser una representación válida del movimiento, y en estas condiciones el movimiento se convierte en inarmónico. Puesto que la magnitud de $\sin \theta$ siempre es menor que la del ángulo $\theta$, la aceleración del péndulo real será notablemente menor que

$$
\frac{d^{2} \theta}{\pi^{2} T^{2}}=-w_{0}{ }^{2} \theta
$$

(válida para ángulos pequeños donde $\sin \ell \triangleq \hat{\theta}$ ) y que corresponde al movimiento armónico angular simple. Por esta razón, una vez que el movimiento se hace apreciablemente inarmónico o anarmónico el periodo ya no sigue constante sino que se alarga cada vez más a medida que aumenta la amplitud, como se mostrará a continuación. Retomando la ecuación (6) la escribimos de la siguiente forma

$$
\left(\cos \theta-\cos \theta_{0}\right)^{-1 / 2} d \theta=\sqrt{\frac{z_{g}}{t}} d t
$$

Haciendo ahora $\theta(t=0)=0$ e integrando desde 0 a $\theta_{0}$ obtenemos,

$$
\int_{0}^{\theta_{2}}\left(\cos \theta-\cos \theta_{0}\right)^{-\frac{2}{2}} d \theta=\sqrt{\frac{2 g}{2}} t
$$

Debe tenerse en cuenta que el tiempo es la cuarta parte de un ciclo completo, es decir la cuarta parte del periodo $T=4 t \quad y$ si consideremos la substitución

$$
\sin \left(\frac{\theta}{2}\right)=\sin \left(\frac{\theta_{\varphi}}{2}\right) \sin (\varphi) \text {, la ecuación (26) se }
$$

reescribe:

$T=4 \sqrt{\frac{2}{g}} \int_{0}^{\frac{\pi}{2}}\left(1-\sin ^{2}\left(\frac{\theta_{c}}{2}\right) \sin ^{2} \varphi\right)^{-1 / 2} d \varphi$

La integral de la anterior ecuación, es una integral elíptica completa de primera clase la cual puede desarrollarse en serie de potencias, quedando como

$T=2 \pi \sqrt{\frac{1}{2}}\left(1+\frac{1}{4} \sin ^{2} \frac{\theta_{0}}{2}+\frac{9}{64} \sin ^{4} \frac{\theta_{0}}{2}+\cdots\right)$

Se obtiene así el valor exacto del periodo en función de la amplitud angular inicial para el péndulo simple. Para ángulos pequeños, los términos de segundo orden no contribuyen significativamente a la suma y se obtiene así el valor conocido para periodo:

$$
T_{0} \approx 2 \pi \sqrt{\frac{1}{g}}
$$

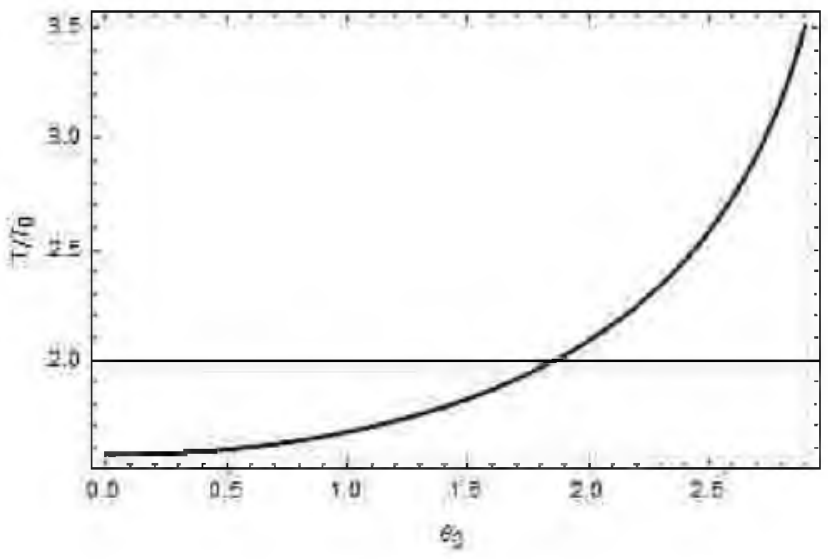

Figura 3. Variación del periodo de un péndulo en función de la amplitud, ecuación (28).

Se observa en la figura 3 , que el periodo $T$ difiere apreciablemente de $T_{0}$ solamente para amplitudes muy grandes. Para pequeñas amplitudes es suficiente tomar el primer término correctivo, aproximación suficiente para la mayor parte de las situaciones prácticas.

A continuación, graficamos la velocidad angular dada por la ecuación (24)

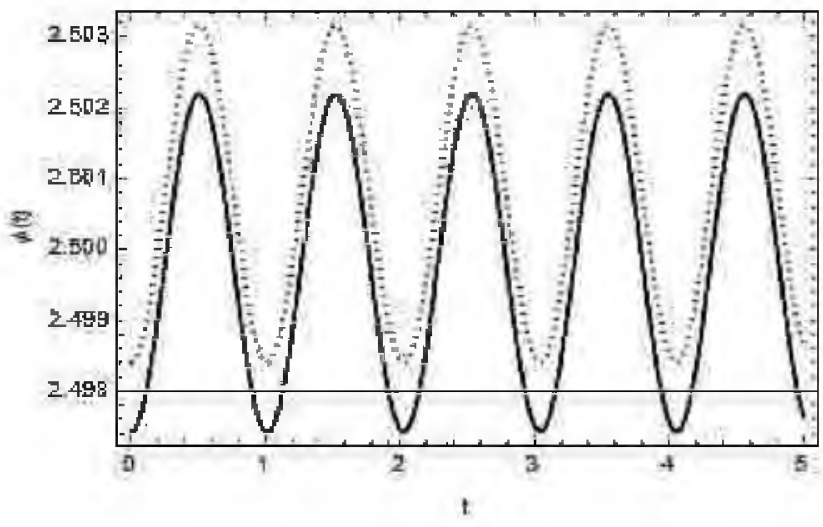

Figura 4. Velocidad angular (24) para velocidad angular inicial de $0 \mathrm{rad} / \mathrm{s}$ (línea continua) y para 4 $\mathrm{rad} / \mathrm{s}$ (línea a trazos).

\section{Conclusiones}

En este trabajo se presentó la solución a la ecuación diferencial no lineal que describe las oscilaciones del péndulo simple, encontramos que tanto el desplazamiento angular, velocidad angular y su periodo, son expresadas en términos de integrales elípticas. Estos resultados se resuelven numéricamente y encontramos que a diferencia de la aproximación frecuentemente utilizada en los textos de física, donde se hace valida la aproximación para ángulos de desplazamiento pequeños sirn $\triangleq$ cuya ecuación diferencial de movimiento se reduce a $\frac{d^{2} \underline{a}}{t^{2}}=-w_{\overline{0}}^{2} \theta$. 
El periodo de oscilación del péndulo es constante e independiente del desplazamiento angular inicial solo para valores pequeños, como se muestra en la figura 3. A medida que el desplazamiento angular inicial aumenta, el periodo de oscilación aumenta considerablemente. Esto es, el movimiento se convierte en inarmónico.

Los resultados presentados pretenden además acercar al estudiante de física y áreas afín, en la introducción de las integrales elípticas y motivar la búsqueda de nuevas alternativas que ofrece la programación para resolver problemas físicos y matemáticos.

\section{Referencias bibliográficas}

Abramowitz, M., y Stegun, A. (1972). Handbook of Mathematical Functions, with formulas, graphs, and mathematical tables. Washington, Estados Unidos: Dover.

Alonso, M., y Finn, E. (1970). Física Volúmen 1 Mecánica. México: Fondo Educativo Interamericano.

Beléndez, A., Pascual, C., Méndez, D., Beléndez, T., and Neipp, C. (2007). Exact solution for the nonlinear pendulum. Revista Brasilera de Ensino de Física, 29, 645-648.

McKelvey, J., y Grotch, H. (1981). Física para ciencias e ingeniería. México: Harla.

Serway, A. (2009). Física para Ciencias e Ingeniería. México: Cengage Learning. 